\title{
Molecular Dynamics as a tool for in silico screening of skin permeability
}

\author{
Paolo Rocco ${ }^{*}$, Francesco Cilurzo $^{\S}$, Paola Minghetti ${ }^{\S}$, Giulio Vistoli ${ }^{\sim}$, Alessandro Pedretti
}

${ }^{\S}$ Department of Pharmaceutical Sciences, Università degli Studi di Milano, via G. Colombo, 71 I-20133 Milan, Italy.

¿Department of Pharmaceutical Sciences, Università degli Studi di Milano, Via Mangiagalli, 25, I-20133 Milan, Italy.

* Corresponding Author. Department of Pharmaceutical Sciences, Università degli Studi di Milano, Via G. Colombo 71, I-20133 Milan, Italy. Phone: +39 (2) 50324656.

E-mail: paolo.rocco@unimi.it.

Keywords

Permeability coefficient, skin permeation, Steered Molecular Dynamics, stratum corneum model, simulation. 


\section{Introduction}

The study of the barrier function of the skin is relevant to a wide range of applications including, in particular, transdermal delivery of drugs and risk assessment of hazardous exposure to chemicals. The skin's barrier capacity, generally ascribed to the outer layer of the skin, the stratum corneum (SC) (Lian et al., 2008), is a function of the molecular organization of the lipids in the extracellular space of the SC (Bouwstra et al., 1991). This lipid matrix is a heterogeneous mixture mainly composed of long-chain ceramides (CERs), free fatty acids (FFAs) - ceramide 2 and FFA 24:0 being the most abundant - and cholesterol (CHOL) in a 1:1:1 molar ratio (Wertz and Norle'n, 2003).

A large number of permeability studies have appeared over the last 60 years, addressing the prediction of skin permeability using mathematical models, in particular semi-empirical or mechanistic models, among which the equations proposed by Potts and Guy (Potts and Guy, 1992) and by Mitragotri (Mitragotri, 2002) are the most cited. Among the physicochemical properties considered as descriptors are the molecular weight and the molecular volume, the octanol/water partition coefficient (Mitragotri, 2002; Potts and Guy, 1992), the molecular radius (Mitragotri, 2002) and the solvation enthalpies (Minghetti et al., 2000).

Along with mathematical modelling, Molecular Dynamics (MD) can be a useful tool to perform permeability studies, with the added benefit that this affords a greater understanding of the permeation process at a molecular level allowing the role of different parts of the SC in determining permeability to be identified. Since the time scales of MD simulations are still orders of magnitude lower than the time scales of the most complex biological events, the atomistic details obtained 
with simulations have to be included in the framework of macroscopic mathematical models (Scott, 2002).

In this work, Steered Molecular Dynamics (SMD) simulations are carried out to investigate the dynamic behaviour of a given permeant when crossing an ideal SC lipid matrix composed of a heterogeneous mixture of long-chain ceramides (CERs), free fatty acids (FFAs), and cholesterol (CHOL) in a 1:1:1 molar ratio. The use of MD simulations to study solute diffusion through lipid bilayer membranes was developed in the 1990s for phospholipid bilayers, starting with early studies on passive diffusion (Alper and Stouch, 1995; Bassolino-Klimas et al., 1995, 1993) moving on to the systematic studies of permeation by Marrink and Berendsen, which took into account diffusion and solubility of permeants into the membrane (Marrink and Berendsen, 1996, 1994), and were then extended to the study of SC permeation by Das and colleagues (Das et al., 2009a, 2009b). The approach used here, though, differs from those of previous works. To allow the screening of 80 molecules, a more indirect method has been attempted, not involving free energy calculations, Instead, only the diffusion coefficient and the conformational space explored by the solute within the different microenvironments inside the SC have been directly derived from the MD simulations.

The performed SMD runs were utilized to derive physicochemical and structural descriptors, while free energy calculations were not considered, especially because such an analysis, using SMD runs and Jarzynski equality (Jarzynski, 1997), would require multiple trajectories for every solute, and this is unfeasible when screening large datasets of permeants. The computed descriptors were correlated to the experimental permeation coefficients contained in a subset of Flynn's set (Flynn, 1990), yielding encouraging predictive models which confirm the fruitfulness of MD simulations to analyse complex biological systems such as the SC at a molecular level. It should 
be noted that any equation obtained by semi empirical models is affected by the experimental error in the database, as better investigated in the Appendix.

\section{Materials and methods}

\subsection{Set-up of the membrane model}

An ideal SC lipid matrix model has been generated according to the model proposed by Iwai et al. (Iwai et al., 2012), which proved to be the most stable upon equilibration. It is composed by a heterogeneous mixture of long-chain ceramides (CERs), free fatty acids (FFAs), and cholesterol (CHOL) in a 1:1:1 molar ratio (Wertz and Norle'n, 2003). Starting with a basic unit composed of one molecule each of CER 2 in extended conformation, 24:0 FFA and CHOL, a minimization by NAMD2 (Phillips et al., 2005) and subsequent optimization by the PM7 semi-empirical method as implemented in MOPAC 2012 (Stewart, 2013, 2012) are performed followed by a $1 \mathrm{~ns}$ molecular dynamics at $300 \mathrm{~K}$. All lipid molecules are in neutral form. 4 basic units (4 CER 2, 4 FFA, 4 CHOL) are assembled to form a monolayer unit. In each monolayer unit, one CER 2 was replaced by one CER 1 (thus giving a $25 \%$ of CER 1 in the ceramide component of the membrane), and the system undergoes a $1 \mathrm{~ns}$ MD simulation after that an energy minimization was performed. Based on this monolayer unit, membrane models of increasing sizes are built, up to a bilayer consisting of 868 lipid molecules (Figure 1a). Then, two layers of $54 \AA$ containing about 8000 water molecules each were added to the model to account for solvation effects in the donor and acceptor phases, and a $10 \mathrm{~ns}$ MD simulation was performed (Figure 1b) to allow for a better relaxation of the bilayer molecules. The MD runs had the same general characteristics as described below. 

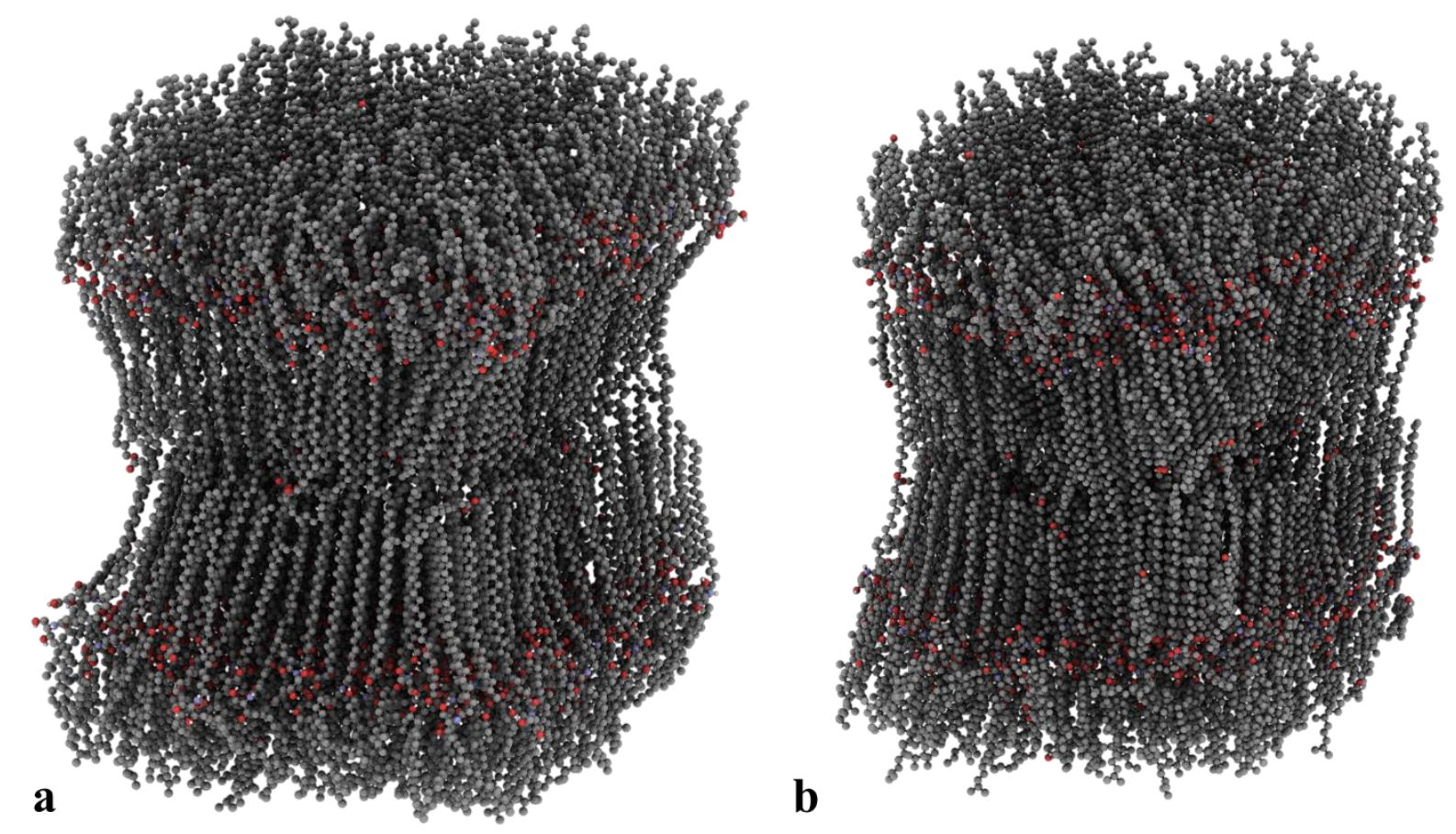

Figure 1. The final 868 molecules bilayer before (a) and after (b) a 10 ns equilibration molecular dynamics in presence of explicit solvent (water, not shown). Apolar hydrogen have been removed from the picture. Images obtained with Qutemol (Tarini et al., 2006).

\subsection{Set-up of the permeants}

The below described SMD simulations were performed on a set of 80 permeants common to both Flynn's set and the Fully Validated set (Supplementary Material), hereinafter named the reduced set. This allowed us to avoid data in Flynn's set that do not meet validation criteria exposed in (Vecchia and Bunge, 2003a), while still being able to compare our results with existing models. In detail, the 80 molecules were simulated in their neutral form since it is reasonably involved in permeation processes. A correction has been applied to the experimental Kp of molecules that are partially ionized at experimental conditions, based on the estimate of the non-ionized fraction (Vecchia and Bunge, 2003a). The conformational profile was explored by a quenched Monte Carlo procedure which produced 1000 conformers by randomly rotating the flexible torsions. For each 
considered permeant, the so obtained lowest energy conformer was further optimized by PM7 semi-empirical calculations (also to derive more precise atomic charges) and underwent SMD simulation.


Figure 2. The solute (circled in red) SMD path through the bilayer before. Snapshots taken every 0.5 ns. Images obtained with Qutemol (Tarini et al., 2006).

\subsection{Steered MD (SMD) simulations}

As a preamble, it should be emphasized that the limited time period under investigation does not allow the spontaneous transport of molecules across the membrane to be simulated. Instead, 
steered molecular dynamics (SMD) simulations are used, where solute molecules are dragged from water into and through the bilayer at a constant velocity in the direction perpendicular to the bilayer surface. As depicted in Figure 2, the permeant is initially placed in the water layer at $69 \AA$ above the bilayer's mid plane and the so obtained system underwent a preliminary minimization to optimize the relative position of the molecules. SMD runs were performed on the 80 permeants with the following characteristics: (a) periodic boundary conditions (108 $\AA$ x $108 \AA$ x $162 \AA$ ) were applied to stabilize the simulation space; (b) Newton's equation was integrated using the r-RESPA method (every 4 fs for long-range electrostatic forces, 2 fs for short-range non-bonded forces, and $1 \mathrm{fs}$ for bonded forces); (c) the long-range electrostatic potential was computed by the Particle Mesh Ewald summation method $(108 \times 108 \times 160$ grid points $)$, the chosen cut-off length was 12 $\AA$ for both the Van der Waals and electrostatic potentials, with a switching function starting a $8 \AA$; (d) the temperature was maintained at $300 \pm 10 \mathrm{~K}$ by Langevin's algorithm; (e) Lennard-Jones (LJ) interactions were calculated with a cut-off of $10 \AA$ and the pair list was updated every 20 iterations; (e) a frame was memorized every 3 ps, thus generating 833 frames; and (f) no constraints were imposed to the systems. The simulations were carried out in two phases: an initial period of heating from $0 \mathrm{~K}$ to $300 \mathrm{~K}$ over 300000 iterations ( $300 \mathrm{ps}$, i.e. $1 \mathrm{~K} / \mathrm{ps}$ ) and the monitored phase of $2.5 \mathrm{~ns}$. During this time, the solute molecule was forced to cover a distance of $50 \AA$ at a speed of $0.02 \AA /$ ps by applying a harmonic constraint force equal to $5 \mathrm{kcal} / \mathrm{mol} / \AA^{2}$.

The 2.5 ns SMD runs were performed using NAMD 2.10 and generally completed in $30 \div 32$ hours depending on the solute, on a Microsoft Windows ${ }^{\circledR}$ PC assembled with off the shelf components, including 1 Xeon $^{\circledR}$ 6-core E5-2620v2 processor and 1 Nvidia ${ }^{\circledR}$ GeForce GTX 780 graphic card. The force fields used are CHARMM 36 (Klauda et al., 2010) for the lipids, and the SPC potential (Berendsen et al., 1981) for water. In the CHARMM force field all atoms are 
described explicitly. CHARMM parameters for lipids, optimized on the condensed phase properties of alkanes (Siu et al., 2008), were introduced in CHARMM22 (Feller et al., 1997), and are periodically updated.

Notably, the trajectory imposed on the solute in the SMD simulations, in which the permeant is made to follow the transversal route through the polar head groups, doesn't necessarily follow the same tortuous path as in the physical system (Mitragotri, 2002), which should instead involve the least dense region. Nevertheless, the simulated trajectory allows for an investigation of the behaviour of the permeants along the whole double layer, through all the possible microenvironments, without prejudice about which one is the path of least resistance, thus taking into account the entire conformational and physicochemical property space (Vistoli et al., 2005) which a permeant explores when moving through the bilayer.

With regard to physicochemical properties, polar surface area (PSA), surface, and Virtual log P as computed by MLP approach (Gaillard et al., 1994) were considered by averaging them in the different zones of the bilayer. To avoid the issues involved with filtering the data from MD simulations, the median of the raw data was used instead of the average. The automatic linear regression script in VEGA ZZ (Pedretti et al., 2002) was used to develop correlations between the physicochemical properties and the permeation coefficient for the set of solutes.

Other descriptors that can be derived directly from the SMD simulations, such as the force acting on the solute molecule, have proved of little value. Improvements for the estimation of the molecule behaviour, such as Free Energy calculations (Marrink and Berendsen, 1994) could not be applied here, since they would require multiple trajectories for every solute, and this is in 
contrast with the primary objective of the study, which is intended as a screening methodology for large sets of molecules.

\section{Results and discussion}

\subsection{Theoretical background}

The flux $\mathrm{J}$ of a given solute across a homogeneous membrane can be written as:

$$
\mathrm{J}=K \cdot \mathrm{D} \cdot \Delta C / \mathrm{h}
$$

where $K$ is the water/membrane partition coefficient, D the diffusion coefficient, $\Delta \mathrm{C}$ the concentration gradient of the solute molecule across the membrane and $\mathrm{h}$ the membrane thickness.

Even though the SC is a non-homogeneous anisotropic medium, and the route of the permeants is a combination of motions running parallel and perpendicular to the bilayer's normal (Mitragotri, 2003), Eq. 1 may represent a simplified approach to the description of the flux across SC. The quantity h, though, should be considered not as the SC thickness, but as the effective path length of the solute inside the SC, which can be expressed as the product of tortuosity, $\tau$ and membrane thickness, $\delta$, namely $\mathrm{h}=\tau \cdot \delta$ (Mitragotri, 2003). By replacing the water/SC partition coefficient $K$ with the octanol/water partition coefficient $P$, the permeation coefficient $\mathrm{Kp}$ can be defined as the flux normalized by the concentration gradient: 


$$
K p=\frac{J}{\Delta C}=\frac{P D}{h}
$$

Eq. 2

The structure of Eq. 2 reveals a first theoretical limit of the semi empirical methods based upon it. Experimental values of $\mathrm{Kp}$ are calculated measuring the flux $\mathrm{J}$ and the concentration gradient $\Delta \mathrm{C}$, whereas theoretical predictions are based on estimation of the quantity $\mathrm{PD} / \mathrm{h}$. An issue arises since the stratum corneum thickness $\delta$, and hence h, may not only vary in different experiments, as well as $\mathrm{pH}$ and temperature, but is also unknown, representing an unavoidable source of variability in experimental data. If $\delta$ values were known for each $\mathrm{Kp}$ measurement, variability would be reduced and only estimates of the tortuosity $\tau$ would be necessary.

In logarithm form, Eq. 2 becomes:

$$
\log K p=\log (P D / h)=\log (P D)-\log h=\log (P D)-\log \tau-\log \delta
$$

As mentioned in the Introduction, the diffusion coefficient $\mathrm{D}$, the octanol/water partition coefficient $\mathrm{P}$ and the path length $\mathrm{h}$ were here derived, directly or indirectly, from the performed SMD simulations. The local diffusion coefficient $D(z)$ can be calculated in different ways from Molecular Dynamics trajectories, the simplest method being based on the mean square 
displacement (MSD). For diffusion in the $z$ direction (but the reasoning can be extended to calculation in the $x y$ plane, $\left.\mathrm{D}_{\mathrm{xy}}\right)$ :

$$
D(z)=\lim _{t \rightarrow \infty} \frac{\left\langle(z(t)-z(0))^{2}\right\rangle}{2 t}
$$

where the average \langle\rangle is, theoretically computed over different system replicas but is replaced, for our purposes, by the time average, a procedure that would be rigorous only if the ergodic hypothesis could be verified. $\mathrm{D}_{\mathrm{xy}}$ was calculated from the MSD in the xy plane obtained from the simulations, using the algorithm proposed by Abrams (Abrams, 2015). The octanol-water partition coefficient $\mathrm{P}$ was predicted by using the average Virtual $\log \mathrm{P}$, as calculated during the simulation (so reflecting the conformational fluctuations of molecules during the simulations) as $\mathrm{P}=10^{\text {VirtualLogP }}$.

As pointed out before, $\delta$ is an unknown experimental value, while hypotheses can be made on the value of $\tau$. Mitragotri (Mitragotri, 2003) proposes that the value of $\tau$ depends not only on the structure of the SC, but also on the physicochemical properties of the solute, in particular its radius. We consider here that properties of the solute, such as molecular dimensions and polarity, influence the path taken by the solute inside the SC, along with structural characteristic of the membrane and temperature. Although lipophilicity and molecular size of the permeant, are already taken into account with the term $\log (\mathrm{PD})$, we can tentatively introduce a linear or exponential dependence of $\tau$ on molecular volume (MV), e.g. $\tau=M V^{\zeta}$ in Eq. 3, obtaining the equation: 
where $\mathrm{P}$ is the octanol/water partition coefficient, D the diffusion coefficient, MV the molecular volume, while a value of 1 or 2 is proposed for $\zeta^{\prime}$ for simplicity. Eq. 4 will be used in the subsequent paragraph as a starting point for the development of predictive equations, dropping the term $\log \delta$, since no assumptions can be made about it.

\subsection{Steered Molecular Dynamics simulations}

As a preamble, it should be remembered that the simulated trajectory is not intended to mimic the physical system but was chosen to avoid any biasing assumption on the possible permeation path, thus allowing an extended exploration of the entire conformational and physicochemical property space (Vistoli et al., 2005) the molecule experiences when moving through the bilayer. To this end, the model membrane was subdivided into 18 regions along the $\mathrm{z}$ direction. A thickness of $4 \AA$ was chosen by considering that a region should not be so large that the calculated descriptors (permeability and diffusion coefficients) vary significantly, while being large enough to allow a significant diffusion coefficient to be calculated from the RMSD values. Since the permeant's trajectories is, on average, from $\mathrm{z}=60 \AA$ (measured from the membrane mid plane) to $\mathrm{z}=20 \AA$, the analyses were performed on the above described regions from $\mathrm{z}=59 \AA$ to $\mathrm{z}=21 \AA$ by moving the frame window by $2 \AA$ at a time.

Eq. 4 was then utilized to develop the corresponding predictive models as generated using the descriptors derived from the 18 so considered regions with $\zeta^{\prime}=1$. The reliability of these models 
is represented in Figure 3 which suggests that optimal statistics are obtained when considering the regions corresponding to the water-phospholipids interface. Then they worsen in the regions substantially characterized by the sole phospholipidic heads to somewhat re-improve in the regions where phospholipidic heads and lipid tails coexist, and finally drop in the pure lipidic regions. These results suggest that promising models can be obtained when considering heterogeneous regions containing molecules or moieties of different polarity, while more homogeneous regions appear to be less performing regardless of their polarity. In particular, Figure 3 reveals that the water/bilayer interface is the primary target to be taken into consideration when studying the behaviour of permeants.

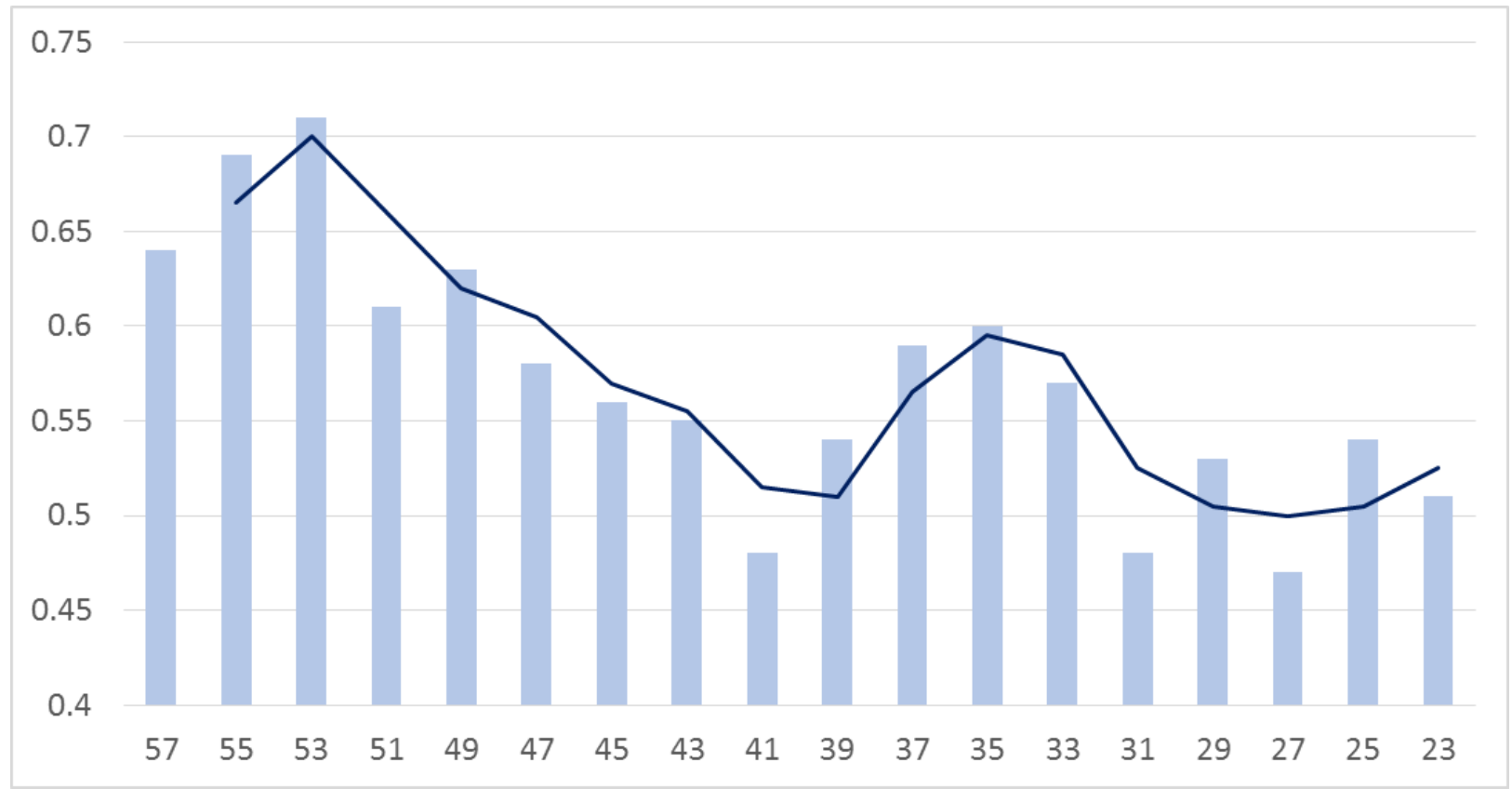

Figure 3. Reliability of the obtained models (expressed by their $\mathrm{r}^{2}$ ) based on Eq.4 and computed using the parameters derived by the 18 different regions in which the in silico membrane was subdivided. 
The so obtained best model is reported in Eq. 5:

$$
\begin{gathered}
\log K p=-1.43+0.72 \log (P D / M V) \\
\mathrm{n}=80 ; \mathrm{r}^{2}=0.71 ; \mathrm{q}^{2}=0.70 ; \mathrm{SE}=0.65 ; \mathrm{F}=193.62
\end{gathered}
$$

where $\mathrm{P}, \mathrm{D}$ and $\mathrm{MV}$ are the octanol/water partition coefficient, the diffusion coefficient and the molecular volume as calculated in region around $z=53 \AA$, respectively.

Such a preliminary equation appears to be promising since it affords results which are roughly comparable to those of the model of Potts and Guy which correlates log Kp to lipophilicity and molecular weight and is one of the most cited semi-empirical equations. Even though the improvement in Eq. 5 seems to be unable to justify the time-demanding use of MD simulations, the beneficial role of the here performed MD simulations is clearly witnessed by the marked worsening of the resulting models in which the MD-derived diffusion coefficients are removed ( $\mathrm{r} 2$ $=0.63$ for the region around $\mathrm{z}=53 \AA$ ) thus inviting further investigations of their predictive role as described below.

As evidenced in Table 3, temperature represents the factor which most markedly influences the variability of the experimental Kp values. Hence, one may hypothesize that improvements on Eq. 5 can be obtained by including parameters which take into account the different temperatures at which the experimental measurements of Kp were performed. In fact, the use of MD runs allows the effects of temperature to be parameterized by two different approaches. In a direct approach, these effects can be considered by performing the simulations at the same temperature as the experiments. This procedure has been avoided so far due to the added computational costs of 
equilibrating the membrane at different temperatures, but will be explored in future studies. In an indirect approach, the effects of temperature can be described by including in the predictive models a specific temperature-dependent parameter as described below.

According to free-volume theory, the dependence of the diffusion coefficient D on absolute temperature T can be expressed by Eq. 6 (strictly valid for small molecules in rubbery polymers) [Errore. Il segnalibro non è definito.]:

$$
D=D_{0} e^{-\gamma M V / T}
$$

Eq. 6

where $\mathrm{MV}$ is the molecular volume, $\mathrm{D}_{0}$ is the diffusion coefficient for $\mathrm{MV} \rightarrow 0$ (constant for a given molecule), and $\gamma$ is a constant. Eq. 6 can be written in logarithm form to give Eq. 6a:

$$
\log D=\log D_{0}-\gamma M V / T
$$

Eq. 6a

Thus and as already pointed out by Vecchia and Bunge (Vecchia and Bunge, 2003a), a term proportional to MV/T (or MW/T where MW is molecular weight) can be used to account for the effect of temperature. Since the simulations were here performed at $300 \mathrm{~K}$, the term $\log \left(\mathrm{PD}_{\mathrm{T}}\right)$ at temperature $\mathrm{T}$ can be expressed in terms of the quantities calculated at $300 \mathrm{~K}$, as 


$$
\log \left(P D_{T}\right)=\log \left(P D_{300}\right)+\gamma M V\left(\frac{1}{300}-\frac{1}{T}\right)=\log \left(P D_{300}\right)+\gamma^{\prime} M V(T-300) / T
$$

Eq. 7

Substituting Eq. 7 for $\log (\mathrm{PD})$ in Eq. 5, the latter assumes the form of Eq. 8

$$
\log K p=\beta_{0}+\beta_{1} \log \left(P D_{300} / M V^{\zeta / \beta_{1}}\right)+\beta_{2} M V \cdot T^{*}
$$

Eq. 8

where $\mathrm{T}^{*}=(\mathrm{T}-300) / \mathrm{T} . \mathrm{P}, \mathrm{D}_{300}$ and $\mathrm{MV}$ are calculated in region around $\mathrm{z}=53 \AA$. It should be noted that the last term in Eq. 8 has the meaning of a correction factor on the experimental log Kp. It equals zero when experimental values of $\mathrm{Kp}$ have been measured at $300 \mathrm{~K}$, being negative when $\mathrm{T}<300 \mathrm{~K}$ and positive when $\mathrm{T}>300 \mathrm{~K}$, in accordance with the average behaviour of residuals (experimental $\operatorname{logKp}$ - predicted $\log \mathrm{Kp}$ ) observed by Vecchia and Bunge (there centred on $30{ }^{\circ} \mathrm{C}$ ) (Vecchia and Bunge, 2003b).

When $\zeta^{\prime}=2$, Eq. 8 becomes:

$$
\log K p=\alpha+\beta \log \left(P D_{300} / M V^{2}\right)+\gamma^{\prime} M V \cdot T^{*}
$$


Although the inclusion of MV in two terms of Eq. 9 may seem questionable, the two terms show a Variance Inflation Factor (VIF) of 1.21, and should be considered as non-collinear independent variables. The coefficients of Eq. 9 can be determined by linear regression analysis, so generating

Eq. 10:

$$
\begin{array}{r}
\log K p=0.21+0.75 \log \left(P D_{300} / M V^{2}\right)+0.066 M V \cdot T^{*} \\
\mathrm{n}=80 ; \mathrm{r}^{2}=0.78 ; \mathrm{q}^{2}=0.77 ; \mathrm{SE}=0.58 ; \mathrm{F}=136.46
\end{array}
$$

Eq. 10

where $\mathrm{P}, \mathrm{D}_{300}$ and MV are calculated in region around $\mathrm{z}=53 \AA$. The scatter plot of Eq. 10 is shown in Figure 4. Notably, the analysis of the residuals evidences that they are greater than 1 only for 8 molecules, and the residual is lower than 0.5 for 51 molecules. Finally, there is no relation between residuals and experimental $\log \mathrm{Kp}$, thus suggesting that Eq. $\mathbf{1 0}$ is similarly predictive for good and poor permeants.

To avoid the double inclusion of the MV variable in the generated equations, a linear dependence on temperature $\mathrm{T}$ can introduced, yielding:

$$
\begin{gathered}
\log K p=-21.21+0.75 \log \left(P D_{300} / M V^{2}\right)+0.072 T \\
\mathrm{n}=80 ; \mathrm{r}^{2}=0.76 ; \mathrm{q}^{2}=0.74 ; \mathrm{SE}=0.61 ; \mathrm{F}=119.94
\end{gathered}
$$


The reported statistics confirm the critical role of the temperature in accounting for the variability of the experimental $\mathrm{Kp}$ values and emphasize that the inclusion of temperature-related parameters yield a notable improvement of the reliability of the so obtained models which seems to justify the time-demanding use of MD simulations.

In order to assess the predictive power of the above reported equations (namely Eqs. 5, 10 and 11), the reduced dataset $(n=80)$ was split into training $(n=53)$ and test $(n=27)$ sets. The correlation equations were generated using only the training set and then used to predict the Kp values for the test set. Such a validation was repeated 20 times randomly subdividing the dataset to render the obtained results reasonably unbiased by the composition of the sets. The correlations for the test set are given in Table 1, while the corresponding statistics for the models generated for the training and test sets are compiled in Table 2. For a comprehensive comparison, Tables 1 and 2 also include the corresponding results as obtained when applying the Potts and Guy equation (shown as P\&G) to the reduced set of permeants as used for Eqs. 5, 10 and 11.

As a trend, Table 1 shows satisfactory relations between experimental and predicted log Kp values in all the reported equations, as assessed by slopes very close to $45^{\circ}$ and intercept values close to 0 . Again, the modest standard deviations emphasize the stability of these equations which are substantially independent on the composition of the test sets.

\begin{tabular}{|c|c|}
\hline Eq. P\&G & $\log K p_{\text {exp }}=0.990( \pm 0.210) \log K p_{\text {pred }}-0.031( \pm 0.473)$ \\
\hline Eq.5 & $\log K p_{\text {exp }}=0.986( \pm 0.172) \log K p_{\text {pred }}-0.017( \pm 0.404)$ \\
\hline
\end{tabular}




\begin{tabular}{|c|c|}
\hline Eq. 10 & $\log \mathrm{Kp}_{\exp }=1.054( \pm 0.141) \log \mathrm{Kp}_{\text {pred }}-0.182( \pm 0.387)$ \\
\hline Eq. 11 & $\log \mathrm{Kp} \mathrm{exp}=0.989( \pm 0.135) \log \mathrm{Kp}_{\mathrm{pred}}-0.006( \pm 0.384)$ \\
\hline
\end{tabular}

Table 1. Equations correlating the predicted $\log \mathrm{Kp}\left(\log \mathrm{Kp} \mathrm{pred}_{\text {) }}\right.$ with the experimental $\log \mathrm{Kp}$ $\left(\log K \mathrm{p}_{\exp }\right)$ for the test sets as derived by utilizing the here reported best equations plus the Potts and Guy equation as a reference. The standard deviation of the coefficients are obtained by considering the 20 equations as computed in this analysis.

\begin{tabular}{|c|c|c|c|c|}
\hline $\begin{array}{c}\text { Equation } \\
(\mathbf{n}=\mathbf{8 0})\end{array}$ & $\begin{array}{c}\mathbf{r}^{2} \\
\text { (training set) }\end{array}$ & $\begin{array}{c}\text { SE } \\
\text { (training set) }\end{array}$ & $\begin{array}{c}\mathbf{r}^{2} \\
\text { (test set) }\end{array}$ & $\begin{array}{c}\text { SE } \\
\text { (test set) }\end{array}$ \\
\hline P\&G & $0.72 \pm 0,04$ & $0.64 \pm 0,04$ & $0.71 \pm 0,08$ & $0.70 \pm 0,08$ \\
\hline Eq. 5 & $0.73 \pm 0.02$ & $0.64 \pm 0.02$ & $0.66 \pm 0.07$ & $0.67 \pm 0.04$ \\
\hline Eq. 10 & $0.79 \pm 0.03$ & $0.58 \pm 0.03$ & $0.75 \pm 0.07$ & $0.57 \pm 0.06$ \\
\hline Eq. 11 & $0.76 \pm 0.02$ & $0.60 \pm 0,04$ & $0.77 \pm 0.05$ & $0.61 \pm 0.08$ \\
\hline
\end{tabular}

Table 2. Statistical parameters $\left(\mathrm{r}^{2}\right.$ and standard error, SE) for training and test set as derived for the best equations plus the Potts and Guy equation as a reference.

The statistics reported in Table 2 confirm that Eq. 5 yields no significant improvements with respect to Potts and Guy equation, while Eq. 10 which takes into account the effect of temperature reveals statistical parameters of both the training and the test sets which are clearly improved compared to both Potts and Guy model and Eq. 5. Notably, the greater simplicity of Eq. 11 
compared to Eq. 10, due to removal of the double inclusion of the volume descriptor, positively impacts on the test sets which show statistics even better than Eq. 10 .

When considering the overall statistics reported in Table 2 and the enhancements in the predictive power exerted by using MD simulations, one may wonder which effect the variability which unavoidably affect all skin permeability data can have on these enhancements. Answering to this basic question can provide a genuine evaluation of the relevance of the improvements gained by Eqs. 10 and 11 and can reveal whether there is still room for improvements, for example, by extracting additional descriptors from MD runs or the reported models are approaching the best achievable predictive power. To this end, the relations between data variability and predictive power are here in depth investigated by targeted numerical simulations as discussed in the Appendix (see below). Notably, such an analysis is here applied to skin permeability data, but the results and the methods reported below have a general applicability.

In detail, the numerical analyses reported in the Appendix estimate how much the maximum achievable $r^{2}$ value decreases when the data uncertainty increases and calculate the corresponding $r^{2}$ confidence interval (see Table A1). Thus, these results can be conveniently applied to the specific case of the here utilized skin permeability data. To get an idea of the typical uncertainty of the pKp values in the existing datasets, compounds for which multiple (more than 2) measurements are included in the FV data set are compiled in Table 3 which reports the standard deviation of $\mathrm{pKp}$ normalized by the pKp average ( $\varepsilon$. The actual value for $\varepsilon$ for the entire Flynn's set or for the Fully Validated data set is unknown. As it can be seen from Table 3, $\varepsilon$ can significantly vary among the reported compounds with a quite large range from 0.03 up to 0.44 . By considering that an intermediate value $(\varepsilon=0.25)$ can represent a fair estimation of the overall 
uncertainty, Table A1 shows that the maximum theoretical value for $\mathrm{r}^{2}$ is 0.74 with a confidence interval of $0.69-0.80$.

This result emphasizes that the here reported equations are really approaching the best theoretically obtainable results for the used experimental Kp values especially considering that the employed descriptors, despite being richly informative, are far from producing an ideal estimator as supposed in the Appendix.

Moreover, the notable correlation $\left(\mathrm{r}^{2}=0.64\right)$ between the normalized standard deviation of experimental $\mathrm{pKp}$ and the normalized standard deviation of experimental temperatures emphasizes the crucial role of temperature which should be taken into consideration to enhance the predictive models for $\mathrm{pKp}$, either in the form of a correction factor (as done here) or performing MD simulations at the same temperature as the experiment. 


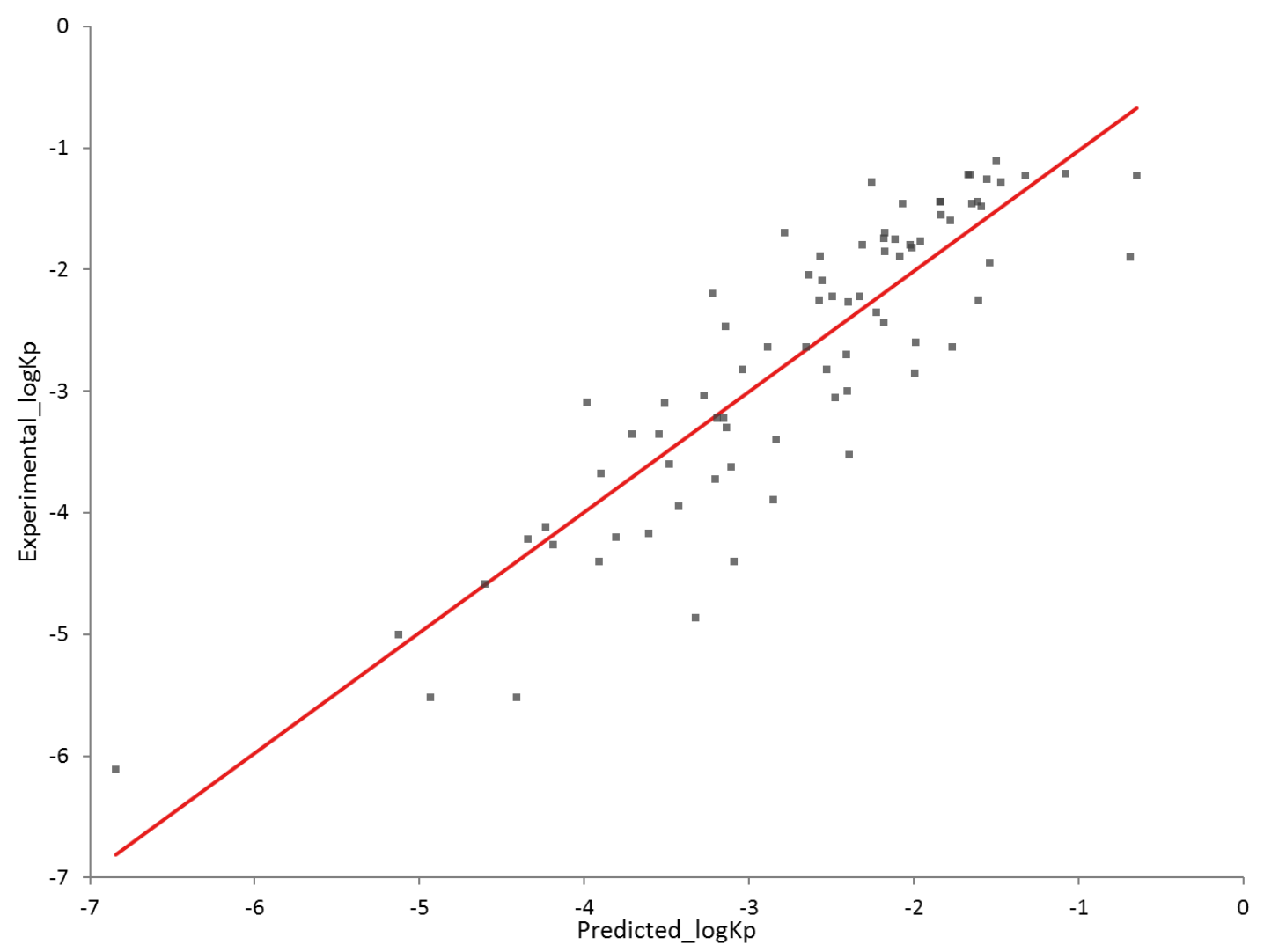

Figure 4. Scatter plot of Eq. 10 showing experimental $\log \mathrm{Kp}$ (x axis) vs predicted log Kp (y axis). 


\begin{tabular}{|lll|}
\hline Corticosterone $(\mathrm{n}=4)$ & $\mathrm{pKp}$ & $\mathrm{t}\left({ }^{\circ} \mathrm{C}\right)$ \\
Average & 3.67 & 29.25 \\
St. Dev. & 0.94 & 6.55 \\
St. Dev./Average $(\varepsilon)$ & 0.26 & 0.22 \\
\hline
\end{tabular}

\begin{tabular}{|lll|}
\hline Phenol $(\mathrm{n}=3)$ & $\mathrm{pKp}$ & $\mathrm{t}\left({ }^{\circ} \mathrm{C}\right)$ \\
Average & 2.53 & 28.00 \\
St. Dev. & 1.12 & 7.94 \\
St. Dev./Average $(\varepsilon)$ & 0.44 & 0.28 \\
\hline
\end{tabular}

\begin{tabular}{|lll|}
\hline Estradiol $(\mathrm{n}=5)$ & $\mathrm{pKp}$ & $\mathrm{t}\left({ }^{\circ} \mathrm{C}\right)$ \\
Average & 2.52 & 31.00 \\
St. Dev. & 0.58 & 4.00 \\
St. Dev./Average $(\varepsilon)$ & 0.23 & 0.13 \\
\hline
\end{tabular}

\begin{tabular}{|lll|}
\hline Ethanol $(\mathrm{n}=3)$ & $\mathrm{pKp}$ & $\mathrm{t}\left({ }^{\circ} \mathrm{C}\right)$ \\
Average & 3.37 & 25.67 \\
St. Dev. & 0.24 & 4.04 \\
St. Dev./Average $(\varepsilon)$ & 0.07 & 0.16 \\
\hline
\end{tabular}

\begin{tabular}{|lcc|}
\hline Mannitol $(\mathrm{n}=4)$ & $\mathrm{pKp}$ & $\mathrm{t}\left({ }^{\circ} \mathrm{C}\right)$ \\
Average & 4.09 & 31.50 \\
St. Dev. & 0.12 & 5.20 \\
St. Dev./Average $(\varepsilon)$ & 0.03 & 0.16 \\
\hline
\end{tabular}

\begin{tabular}{|lll|}
\hline Water $(\mathrm{n}=8)$ & $\mathrm{pKp}$ & $\mathrm{t}\left({ }^{\circ} \mathrm{C}\right)$ \\
Average & 2.96 & 29.88 \\
St. Dev. & 0.20 & 2.10 \\
St. Dev./Average $(\varepsilon)$ & 0.07 & 0.07 \\
\hline
\end{tabular}

\begin{tabular}{|lcc|}
\hline Salicylic acid $(\mathrm{n}=3)$ & $\mathrm{pKp}$ & $\mathrm{t}\left({ }^{\circ} \mathrm{C}\right)$ \\
Average & 1.72 & 34.67 \\
St. Dev. & 0.43 & 4.04 \\
St. Dev./Average $(\varepsilon)$ & 0.25 & 0.12 \\
\hline
\end{tabular}

\begin{tabular}{|lll|}
\hline Octanol $(\mathrm{n}=3)$ & $\mathrm{pKp}$ & $\mathrm{t}\left({ }^{\circ} \mathrm{C}\right)$ \\
Average & 1.26 & 25.67 \\
St. Dev. & 0.04 & 4.04 \\
St. Dev./Average $(\varepsilon)$ & 0.03 & 0.16 \\
\hline
\end{tabular}

Table 3. Average, Standard Deviation (St. Dev.) and St. Dev. normalized by average for experimental $\mathrm{pKp}$ and temperature $\left(\mathrm{t},{ }^{\circ} \mathrm{C}\right)$ based on multiple data for the same compound included in the Fully Validated data set (Vecchia and Bunge, 2003a). 


\section{CONCLUSIONS}

After building a stable and equilibrated model for the stratum corneum lipid bilayer, steered molecular dynamics simulations have been performed using molecules in the common subset between Flynn's set and the Fully Validated set as permeants. The equation obtained compares well with the Potts and Guy equation.

Molecular dynamics has been used in an indirect way as a mean to explore the conformational and property space of the solute molecules in the different microenvironments of the stratum corneum. Since the work was meant to be a first screening of a large set of solutes, the approach developed (for plasma membranes) by Marrink and Berendsen for the prediction of permeability was not applied due to the added computational cost of Free Energy calculations from SMD simulations for 80 permeants. Instead, a mixed approach could be used where only the averaged diffusion coefficient in the plane parallel to the bilayer surface is extracted by MD runs by using the method of Marrink and Berendsen, and then used together with the physicochemical properties in the correlative equation. It should be noted that, due to the parallel nature of the task, running multiple simultaneous simulations (on the same solute to perform Free Energy calculations, on different solutes to cover a larger data set) is possible and only a matter of dedicating additional processing units to the job.

However, the considered approach has allowed us to correlate the permeability coefficient to averaged (or median) physicochemical properties, improving on existing semi-empirical methods (employing the same quantities determined experimentally). Further improvement of the correlation coefficient seems difficult, considering the heterogeneity of the experimental dataset and the error involved in the measurement of the permeability coefficients. As a matter of fact, the statistics of the generated equations are unavoidably affected by the average error included in the 
experimental data and this poses the problem concerning the optimal $\mathrm{r}^{2}$ value which can be reached starting from a given set of experimental data. Since such a problem particularly affects the prediction of the transdermal permeation, it was in depth treated in the following appendix by taking into consideration the here utilized dataset, even though the obtained results are of general applicability.

Further collection of SMD data, may still allow us to gain a better understanding of the physical process. In detail, the behaviour of the molecules in the different zones of the bilayer may help to clarify whether the dependency of permeation upon molecular weight is due to a different region involved as a limiting step during the permeation process. 


\section{APPENDIX}

\section{Maximum theoretical value and confidence interval of the correlation coefficient $r$}

When there is great variability in the compiled experimental dataset, considerations on the confidence interval of $\mathrm{r}$ and on the maximum theoretical value achievable for $\mathrm{r}$ can offer interesting results as to what to expect from a predictive model based on that set. In this appendix, purposely designed numerical simulations will be utilized to reveal how much the $r$ value is worsened by increasing the data uncertainty and then the corresponding confidence interval of $\mathrm{r}$ will be determined by using the Fisher $\mathrm{r} \rightarrow \mathrm{Z}$ transform (Fisher, 1936, 1921, 1915).

Thus, we can assume that a perfect estimator $\varphi$ for a set of experimental data $\mathrm{y}_{\mathrm{i}}$ is known (in our study $y_{i}$ would correspond to the here utilized $\log \mathrm{Kp}$ values). The estimator $\varphi$ is a mathematical function which correlates a set of variables $\left\{\mathrm{x}_{\mathrm{ij}}\right\}$ with the experimental value $\mathrm{y}_{\mathrm{i}}$, where $\mathrm{x}_{\mathrm{ij}}$ represents the j-th molecular property of the i-th molecule (see Eq. A1). The correlation based on a perfect estimator yields a correlation coefficient $r=1$.

$$
\mathrm{y}_{\mathrm{i}}=\varphi\left(\mathrm{x}_{\mathrm{ij}}\right) \quad \mathrm{i}=1,2 \ldots \mathrm{n}, \mathrm{j}=1,2 \ldots
$$

For every $\mathrm{y}_{\mathrm{i}}$, we now introduce an error equal to $\varepsilon \cdot \mathrm{c}_{\mathrm{i}} \cdot \mathrm{y}_{\mathrm{i}}$, where the $\mathrm{c}_{\mathrm{i}}$ values are normally distributed pseudo-random numbers with zero average and unitary standard deviation as calculated by applying the Box-Muller transform to a set of a linearly distribute random numbers (Box et al., 1958), and $\varepsilon$ corresponds to the standard deviation of the errors, normalized by $\mathrm{y}_{\mathrm{i}}$. On this basis, Eq. A1 becomes Eq. A2 which has necessarily a correlation coefficient $r<1$. 


$$
\mathrm{yi}_{\mathrm{i}}\left(1 \pm \varepsilon \mathrm{c}_{\mathrm{i}}\right)=\varphi\left(\mathrm{x}_{\mathrm{ij}}\right) \quad \mathrm{i}=1,2 \ldots \mathrm{n}, \mathrm{j}=1,2 \ldots
$$

Since $\varphi$, by definition, is a perfect estimator, the $r$ values obtained in the simulation are the maximum theoretical correlation coefficients achievable given the uncertainty introduced ( $(\boldsymbol{\varepsilon})$.

For different values of $\varepsilon$, we repeat the numerical simulation 99 times so calculating the 99 correlation coefficients $\mathrm{r}_{\mathrm{k}}$, as obtained by applying Eq. A2 99 times to the same set $\left\{\mathrm{y}_{\mathrm{i}}, \mathrm{x}_{\mathrm{ij}}\right\}$, each time changing the set $\left\{\mathrm{c}_{i}\right\}$. Table A1 clearly shows how much $\mathrm{r}$ and $\mathrm{r}^{2}$ worsen when $\varepsilon$ increases and confirms that the formula maximum $r^{2} \cong 1-\varepsilon$ is an approximate but yet reasonable way to quickly estimate the worsening effect of $\varepsilon$.

After determining the maximum $r$ value, the confidence interval of $r$ can be estimated by using the Fisher $\mathrm{r} \rightarrow \mathrm{Z}$ transform. It is defined by $\mathbf{E q}$. $\mathbf{A 3}$ as:

$$
Z=\frac{1}{2}[\ln (1+r)-\ln (1-r)]
$$

The transformed variable $\mathrm{Z}$ has a different behaviour than $\mathrm{r}$. It tends to a normal distribution as the number of data (n) becomes large. To get a confidence interval for $r$, the value of $r$ is transformed into $\mathrm{Z}$, a confidence interval is calculated for $\mathrm{Z}$ and then is transformed in the corresponding confidence interval of $r$, using the reverse transform Eq. A4: 


$$
r=\frac{e^{2 Z}-1}{e^{2 Z}+1}
$$

Eq. A4

Since the previously calculated $r_{k}$ values are not normally distributed, we apply Fisher $r \rightarrow Z$ transform and obtain the corresponding $99 \mathrm{Z}_{\mathrm{k}}$ values, for which the standard deviation is calculated by Eq. A5:

$$
S_{z}=\frac{1}{99} \sum_{k=1}^{99}\left(Z_{k}-\bar{Z}\right)^{2}
$$

Once the confidence interval for $Z$, has been calculated as $\left(\bar{Z}-S_{z}, \bar{Z}+S_{z}\right)$, the reverse transform (Eq. A4) is used to derive a confidence interval for r. Results of the simulations (Table A1) show how the confidence interval of $r$ enlarges when the data uncertainty increases and allows a reasonable estimation of the range in which $\mathrm{r}$ can fallow depending of the data variability while considering that these simulations are based on an ideal situation in which a perfect estimator is available. 


\begin{tabular}{|l|l|l|l|l|l|l|}
\hline \multicolumn{1}{|c|}{$\varepsilon \rightarrow$} & 0.1 & 0.15 & 0.2 & 0.25 & 0.3 & 0.4 \\
\hline Maximum average $r$ & 0.973 & 0.94 & 0.90 & 0.86 & 0.82 & 0.73 \\
\hline Confidence interval for $r$ & $0.968-0.979$ & $0.93-0.96$ & $0.88-0.83$ & $0.83-0.89$ & $0.77-0.86$ & $0.66-0.79$ \\
\hline Maximum average $r^{2}$ & 0.948 & 0.89 & 0.82 & 0.74 & 0.67 & 0.53 \\
\hline${\text { Confidence interval for } r^{2}}^{2}$ & $0.936-0.959$ & $0.87-0.91$ & $0.78-0.86$ & $0.69-0.80$ & $0.60-0.74$ & $0.44-0.63$ \\
\hline
\end{tabular}

Table A1. Maximum theoretical average values and confidence interval for $r$ and $r^{2}$ obtained through numerical simulation, corresponding to different values of $\varepsilon=$ (Std. dev. of experimental $p K p) /($ average experimental $p K p$ ) in a set of measurements for the same compound. 
CONFLICTS OF INTEREST

The Authors declare that they have no conflicts of interest. 


\section{REFERENCES}

Abrams, C., 2015. Molecular Simulations. Case Study 3: Dynamical Properties: The SelfDiffusion Coefficient. URL http://www.pages.drexel.edu/ cfa22/msim/node29.html

Alper, H.E., Stouch, T.R., 1995. Orientation and diffusion of a drug analogue in biomembranes: Molecular dynamics simulations. J. Phys. Chem. 99, 5724-5731.

Bassolino-Klimas, D., Alper, H.E., Stouch, T.R., 1993. Solute diffusion in lipid bilayer membranes: an atomic level study by molecular dynamics simulation. Biochemistry 32, 1262412637.

Bassolino-Klimas, D., Alper, H.E., Stouch, T.R., 1995. Mechanism of solute diffusion through lipid bilayer membranes by molecular dynamics simulation. J. Am. Chem. Soc. 117, 4118-4129.

Berendsen, H., Postma, J., van Gunsteren, W., Hermans, J., Pullman, B., 1981. Interaction models for water in relation to protein hydration., in: Pullman B (Ed.), Intermolecular Forces. Reidel, Dordrecht, pp. 331-342.

Bouwstra, J.A., Gooris, G.S., van Bras, W., der Speck, J.A., 1991. Structural investigations of human stratum corneum by small-angle X-ray scattering. J. Invest. Dermatol. 97, 1005-1012.

Box, G.E.P., Mervin, E., Muller, A., 1958. Note on the Generation of Random Normal Deviates. Ann. Math. Stat. 29, 610-611.

Das, C., Olmsted, P.D., Noro, M.G., 2009. Simulation studies of stratum corneum lipid mixtures. Biophys. J. 97, 1941-1951.

Das, C., Olmsted, P.D., Noro, M.G., 2009. Water permeation through stratum corneum lipid bilayers from atomistic simulations. Soft Matter 5, 4549-4555. 
Feller, S.E., Yin, D., Pastor, R.W., MacKerell, A.D.J., 1997. Molecular dynamics simulation of unsaturated lipid bilayers at low hydration: parameterization and comparison with diffraction studies. Biophys. J. 73, 2269-2279.

Fisher, R.A., 1936. Statistical Methods for Research Workers. Oliver and Boyd, Edimburgh.

Fisher, R.A., 1915. Frequency distribution of the values of the correlation coefficient in samples of an indefinitely large population. Biometrika 10, 507-521.

Fisher, R.A., 1921. On the "probable error" of a coefficient of correlation deduced from a small sample. Metron 1, 3-32.

Flynn, G.L., 1990. Physicochemical determinants of skin absorption., in: Garrity, T.R., Henry, C.J. (Eds.), Principles of Route to Route Extrapolation for Risk Assessment. Elsevier, New York, pp. 93-127.

Gaillard, P., Carrupt, P.A., Testa, B., Boudon, A., 1994. Molecular Lipophilicity Potential, a tool in 3D QSAR: Method and applications. J. Comput. Aided. Mol. Des. 8, 83-96.

Iwai, I., Han, H., Hollander, L., Svensson, S., Ofverstedt, L.G., Anwar, J., Brewer, J., Bloksgaard, M., Laloeuf, A., Nosek, D., Masich, S., Bagatolli, L.A., Skoglund, U., den Norlén, L., 2012. The human skin barrier is organized as stacked bilayers of fully extended ceramides with cholesterol molecules associated with the ceramide sphingoid moiety. J. Invest. Dermatol. 132, 2215-2225.

Jarzynski, C., 1997. Nonequilibrium Equality for Free Energy Differences. Phys. Rev. Lett. 78, 2690-2693. 
Klauda, J.B., Venable, R.M., Freites, J.A., O’ Connor, J.W., Tobias, D.J., Mondragon-Ramirez, C., Vorobyov, I., MacKerell, A.D.J., Pastor, R.W., 2010. Update of the CHARMM all-atom additive force field for lipids: Validation on six lipid types. J. Phys. Chem. B 114, 7830-7843.

Lian, G., Chen, L., Han, L., 2008. An evaluation of mathematical models for predicting skin permeability. J. Pharm. Sci. 97, 584-598.

Marrink, S.J., Berendsen, H.J.C., 1994. Simulation of water transport through a lipid membrane. J. Phys. Chem. 98, 4155-4168.

Marrink, S.J., Berendsen, H.J.C., 1996. Permeation process of small molecules across lipid membranes studied by molecular dynamics simulations. J. Phys. Chem. 100, 16729-16738.

Minghetti, P., Casiraghi, A., Cilurzo, F., Montanari, L., Monzani, M. V, Bertolini, G., Zaliani, A., 2000. Solvation enthalpies as descriptors of structure-in vitro percutaneous permeation relationship of benzoxazinones regioisomers. Farmaco 55, 563-568.

Mitragotri, S., 2002. A theoretical analysis of permeation of small hydrophobic solutes across the stratum corneum based on Scaled Particle Theory. J Pharm Sci 91, 744-752.

Mitragotri, S., 2003. Modeling skin permeability to hydrophilic and hydrophobic solutes based on four permeation pathways. J. Control. Release 86, 69-92.

Pedretti, A., Villa, L., Vistoli, G., 2002. Vega: a versatile program to convert, handle and visualize molecular structure on Windows-based PCs. J. Mol. Graph. 21, 47-49.

Phillips, J.C., Braun, R., Wang, W., Gumbart, J., Tajkhorshid, E., Villa, E., Chipot, C., Skeel, R.D., Kale, L., Schulten, K., 2005. Scalable molecular dynamics with NAMD. J. Comput. Chem. $26,1781-1802$. 
Potts, R.O., Guy, R.H., 1992. Predicting skin permeability. Pharm. Res. 9, 663-669.

Scott, H.L., 2002. Modeling the lipid component of membranes. Curr. Opin. Struct. Biol. 12, 495-502.

Siu, S.W., Vácha R, Jungwirth P, Böckmann RA, 2008. Biomolecular simulations of membranes physical properties from different force fields Vácha R. J. Chem. Phys. 128, 125103.

Stewart, J.J.P., 2013. Optimization of parameters for semiempirical methods VI: more modifications to the NDDO approximations and re-optimization of parameters. J. Mol. Model. 19, $1-32$.

Stewart, J.J.P., 2012. MOPAC2012. Stewart Computational Chemistry; Colorado Springs, CO, USA. 2012, HTTP://OpenMOPAC.net

Tarini, M., Cignoni, P., Montani, C., 2006. Ambient occlusion and edge cueing to enhance real time molecular visualization. IEEE Trans. Vis. Comput. Graph. 6.

Vecchia, B.E., Bunge, A.L., 2003. Skin Absorption Databases and Predictive Equations., in: Guy R.H., H.J. (Ed.), Transdermal Drug Delivery. CRC Press, New York, pp. 100-106.

Vecchia, B.E., Bunge, A.L., 2003. Evaluating the Transdermal Permeability of Chemicals., in: Guy RH, H.J. (Ed.), Transdermal Drug Delivery. CRC Press, New York, pp. 38-39.

Vistoli, G., Pedretti, A., Villa, L., Testa, B., 2005. Solvent constraints on the property space of acetylcholine. I Isotropic solvents. J. Med. Chem. 48, 1759-1767. 
Wertz, P., Norle'n, L., 2003. "Confidence Intervals" for the "true" lipid compositions of the human skin barrier?, in: Forslind, B., Lindberg, M. (Eds.), Skin, Hair, and Nails Structure and Function. Marcel Dekker, New York, pp. 85-106. 